\title{
Miranda
}

Revue pluridisciplinaire du monde anglophone /

Multidisciplinary peer-reviewed journal on the English-

speaking world

$14 \mid 2017$

Early American Surrealisms, 1920-1940 / Parable Art

\section{AS - Le blues et le diable font bon ménage}

\section{Patrice Larroque}

\section{OpenEdition}

\section{Journals}

Édition électronique

URL : http://journals.openedition.org/miranda/10186

DOI : 10.4000/miranda.10186

ISSN : 2108-6559

\section{Éditeur}

Université Toulouse - Jean Jaurès

\section{Référence électronique}

Patrice Larroque, « AS - Le blues et le diable font bon ménage », Miranda [En ligne], 14 | 2017, mis en ligne le 18 avril 2017, consulté le 16 février 2021. URL : http://journals.openedition.org/miranda/10186 ; DOI : https://doi.org/10.4000/miranda.10186

Ce document a été généré automatiquement le 16 février 2021.

\section{c) 98}

Miranda is licensed under a Creative Commons Attribution-NonCommercial-NoDerivatives 4.0 International License. 


\title{
AS - Le blues et le diable font bon ménage
}

\author{
Patrice Larroque
}

1 Les origines exactes de la musique que l'on appelle aujourd'hui le blues se perdent un peu dans le temps. Il est en réalité difficile de lui donner une date de naissance précise à cause justement de son évolution qui s'étale sur une assez longue période. Il existait bien avant qu'on lui donne un nom, depuis le début de l'esclavage en Amérique en 1619, dans les chansons que les esclaves noirs entonnaient au moment des travaux des champs et dans les réunions religieuses.

2 Ainsi, l'histoire du blues commence avec l'apparition des premiers esclaves noirs sur le sol américain, mais coupés dès leur arrivée de leurs racines et de leurs langues africaines, ils ont dû recréer de nouvelles attaches en s'appuyant sur une nouvelle langue, l'anglais, qu'ils ont adopté et modelé pour en faire la langue du blues. Enfin, le blues possède également une fonction sociale qui a permis à la population noire de survivre, de trouver sa place dans la société américaine, de dépasser les clivages raciaux et de s'émanciper.

\section{Le contexte historique}

\subsection{Les origines}

3 Le blues est donc une forme musicale traditionnelle, d'abord vocale et non écrite, dérivée des work songs et des field hollers, chants et complaintes qui rythmaient le travail et le rendaient moins pénible. Ils apportaient un peu de joie de vivre à la tristesse et à la dureté des conditions de vie des esclaves noirs importés aux Etats-Unis d'Amérique dès le XVIIe siècle pour travailler dans les plantations de coton ou de maïs. Ces chants ont donné naissance au blues rural vers la fin du XIXe siècle dans le sud des Etats-Unis et en particulier dans la région du delta du Mississippi.

4 A l'origine du blues on trouve aussi le negro spiritual. C'est une forme de chant religieux issu des camp meetings, sorte d'assemblées en plein air à vocation religieuse qui se sont 
développées au début du XIXe siècle. Les rites ancestraux des esclaves noirs, interdits par les maitres et souvent punis du fouet ou de mort, ont peu à peu laissé la place à la religion chrétienne que leur enseignaient les prêcheurs américains. Les hymnes et les cantiques chrétiens étaient adaptés au caractère musical hérité de l'Afrique. Ils étaient répétés et appris par cœur, sans vraiment comprendre, et faisait une place importante à l'improvisation. William Faulkner décrit une de ces messes à la fin de son roman Soldier's Pay:

The road dropped on again descending between reddish gashes, and across a level moon-lit space, broken by a clump of saplings, came a pure quivering chord of music wordless and far away.

'They're holding services. Negroes,' the rector explained.

They walked on in the dust, passing neat tidy houses, dark with slumber. An occasional group of negroes passed them, bearing lighted lanterns that jetted vain little flames futilely into the moonlight. 'No one knows why they do that,' the divine replied to Gillian's question. 'Perhaps it is to light their churches with.'

The singing drew nearer and nearer; at last, crouching among a clump of trees beside the road, they saw the shabby church with its canting travesty of a spire. Within it was a soft glow of kerosene serving only to make the darkness and the heat thicker, making thicker the imminence of sex after harsh labour along the mooned land; and from it welled the crooning submerged passion of the black race. It was nothing, it was everything; then it swelled to an ecstasy, taking the white man's words as readily as it took his remote God and made a personal Father of Him.

Feed Thy Sheep, O Jesus. All the longing of mankind for a Oneness with Something, somewhere. Feed Thy Sheep, O Jesus. ... the rector and Gillian stood side by side in the dusty road. The road went on under the moon, vaguely dissolving without perspective. Worn-out red-gutted fields were now alternate splashes of soft black and silver; trees had each a silver nimbus, save those moonward from them, which were sharp as bronze.

Feed Thy Sheep, O Jesus. The voices rose full and soft. There was no organ: no organ was needed as above the harmonic passion of bass and baritone soared a clear soprano of women's voices like a flight of gold and heavenly birds. They stood together in the dust, the rector in his shapeless black, and Gillian in his new hard serge, listening, seeing the shabby church become beautiful with mellow longing, passionate and sad. Then the singing died, fading away along the mooned land inevitable with tomorrow and sweat, with sex and death and damnation; and they turned townward under the moon, feeling dust in their shoes. $(265-266)^{1}$

5 Souvent, les airs et les paroles étaient créés ou modifiés à la mode de la musique populaire traditionnelle des pays de langue anglaise (Angleterre, Canada, Ecosse, EtatsUnis, Irlande), une musique venue d'Europe.

6 Le contact entre les Blancs et les Noirs a sans doute aussi contribué à accélérer la conversion de la population noire au christianisme. La culture religieuse spécifique aux Africains a facilité le passage d'une croyance en plusieurs dieux à une foi religieuse monothéiste occidentale. Cette influence blanche se retrouve également dans la musique du blues.

7 Enfin, les negro spirituals qui exprimaient les sentiments de misère et d'abandon de la population noire ont sans doute influencé les work songs collectifs. Le blues, mélange de profane et de sacré, est dans la tristesse de ces chants. Il décrit une certaine forme de 
mélancolie et de découragement. Aujourd'hui, quand on a du vague à l'âme, qu'on est déprimé, on dit qu'on a le blues; du blues des infirmières à celui des jeunes désœuvrés ou des chômeurs, l'expression a fait son chemin, mais quelle est son origine?

\subsection{Le mot blues}

8 Il semble que l'origine du terme blues soit l'abréviation de l'expression anglaise " the blue devils » qui signifie idées noires, dépression et tristesse. On retrouve une référence au blues ou aux blue devils dans une formule : « ... for having a fit of the blue devils », tirée d'une pièce de théâtre de George Colman le jeune (1762-1836), Blue Devils, a Farce in One Act, publiée en 1798. L'expression était également employée pour désigner le delirium tremens.

9 Aux Etats-Unis, le mot blues est employé pour la première fois dans le journal de Charlotte Forten, une institutrice noire née libre dans le nord et qui a choisi d'alphabétiser des esclaves de Caroline du Sud. Entre 1862 et 1865, elle a tenu un journal ${ }^{2}$ dans lequel elle relate la misère et le malheur qui régnaient dans les quartiers d'esclaves (Herzhaft $2008: 12$ ). Dans la musique noire américaine, le terme apparaît à Memphis vers 1912, date du premier enregistrement de la chanson Memphis Blues par le compositeur W. C. Handy, aujourd'hui considéré comme le père du blues.

Les formes de blues les plus primitives provenaient, répétons-le, du sud des Etats-Unis où étaient employés des esclaves essentiellement originaires d'Afrique occidentale.

\subsection{Les influences africaines}

11 De nombreux traits spécifiques au blues viennent de la musique africaine. On en citera deux en particulier : les mélismes qui sont des phrases ornementales de plusieurs notes chantées sur une seule syllabe du texte et l'intonation ondulante, roulante et nasale.

Parmi les instruments qui rappellent l'Afrique, il y a bien sûr l'omniprésente guitare et le banjo qui remplacent sans doute la kora, instrument à cordes utilisé par certains peuples d'Afrique occidentale, en Gambie par exemple, le banjo étant lui aussi dérivé d'un ou plusieurs instruments de musique africains. Dans son autobiographie, W. C. Handy (1941) fait allusion à un guitariste noir qui, pendant qu'il jouait, appuyait un couteau sur les cordes de sa guitare. Cette technique du couteau n'est pas sans évoquer l'utilisation actuelle du bottle neck (cylindre de verre ou de métal que l'on fait glisser sur les cordes de la guitare) et à la steel guitar (technique qui consiste à tenir l'instrument horizontalement sur ses genoux, les cordes vers le haut, et à faire glisser une barrette d'acier sur les cordes le long du manche). Cette technique du couteau se retrouve, semble-t-il, dans les cultures d'Afrique centrale et occidentale.

Les Africains de l'ouest ne chantaient probablement pas de blues, mais on sait que les premiers esclaves chantaient ou criaient en travaillant et que ces chants ressemblaient beaucoup à ceux de l'Afrique occidentale (Jones 1968 : 14). Ces chants ou ces cris se chantaient a capella, sans accompagnement : le rythme et la mesure étaient donc libres. Peut-être les work songs et les negro spirituals collectifs étaient-ils plus contraints du point de vue de la cadence, mais on peut déjà voir apparaitre un style vocal dans lequel la langue occupe une place privilégiée en tant que support rythmique et mélodique. 


\section{La langue du blues}

\subsection{La forme} et $d u$ jazz, Michel Fabre insiste sur les relations étroites qu'entretiennent langue et musique dans l'expression des Noirs aux Etats-Unis. La langue est celle de la vieille ballade anglaise dont la structure en huit, dix ou seize mesures a servi de base aux chansons de type blues. En réalité, le blues traditionnel est en douze mesures, quatre mesures qui se répètent trois fois, ce qui nous donne seize battements par groupe pour une mesure à quatre temps. Ceci est à rapprocher de l'assertion de Ker $(1928: 208)$ qui soutient que le rythme naturel pour la race humaine roule par périodes de quatre, huit ou seize battements ou groupes de battements, le groupe de quatre battements étant, semble-t-il, le plus courant en poésie populaire anglaise (Attridge 1982:80-82), ce qui équivaut à quatre temps en musique. Mais c'est essentiellement le cri et les chants africains en appels et réponses (call and response shouts) qui ont dicté au blues la forme qu'il a aujourd'hui. Il en a adopté la structure (Jones $1968: 102$ ). les répétions, les reprises, le rythme syncopé et surtout l'absence d'acte de composition qui laisse une large place à l'improvisation, assurent la primauté de la parole, donc du texte et de la langue sur la musique. Le chanteur de blues s'exprime dans l'idiome du blues et s'inscrit dans la tradition orale afro-américaine (Levet 1992: 31-36). Il délivre un message souvent spirituel sous une forme quasi-rituelle (Springer $1999: 19$ ). fois :

(1) Oh, Lawd, I'm tired, uuh Oh Seigneu', j'en ai maah, aah

oh, Lawd, I'm tired, uuh Oh, Seigneu', j'enai maah, aah

oh, Lawd, I'm tired, uuh oh seigneu', j'en ai maah, aah

Oh, Lawd, I'm tired, Oh, Seigneu', j'en ai maah,

a dis mess. d'cette mé'asse. (cité dans Jones $1968: 100$ )

17 Et ce n'est que bien plus tard que la structure actuelle, composée d'un vers repris une fois et suivi d'un vers de conclusion, est devenue la norme :

(2) I followed her to the station with a suitcase in my hand, And I followed her to the station with my suitcase in my hand.

Well it's hard to tell, it's hard to tell when my love's in vain,

My love's in vain.

(R. Johnson, Love in Vain) d'une véritable mélodie.

Il n'est certes pas étonnant que la musique vocale reproduise la prosodie d'un discours qu'elle adapte aux traits dominants, rythmique et mélodique, du texte. Deux vers célèbres (et repris maintes fois) de Robert Charles Guidry, un musicien cajun de Louisiane, illustrent bien ce jeu verbal et musical :

(3) See you later, alligator,

After 'while, crocodile.

Miranda, 14 | 2017 
put entendre les pieds métriques trochaïques qui, associés aux rimes internes, contribuent à rendre la musique de l'anglais. Il est à noter que le déterminant $a$ normalement placé devant le nom while est muet (remplacé dans la graphie par une apostrophe). Le vide accentuel (deux syllabes inaccentuées adjacentes) est ainsi modifié pour obtenir une structure plus eurythmique parallèle au premier vers.

21 Le blues est un art folklorique (Springer 1999: 17), et l'influence linguistique sur le rythme de la musique est d'une manière ou d'une autre guidée par le texte. En revanche, l'idée que les schémas discursifs se reflètent dans la musique instrumentale est beaucoup plus controversée (Patel et al. 2006 : 3034). Cependant, si l'on considère que la musique instrumentale d'une culture peut refléter la prosodie de sa langue maternelle, on peut concevoir l'idée qu'une partie du rythme si spécifique à l'anglais tient à l'alternance des voyelles pleines et des voyelles réduites (Bolinger 1985, Carr 1999). Et il semble que cette tendance se retrouve dans la musique. Ceci peut paraître contradictoire avec le caractère partiellement syllabé de l'anglais afro-américain. Mais comme nous l'avons dit, il ne s'agit que d'une tendance, le rythme globalement accentuel de l'anglais américain prédominant.

\subsection{Le poids linguistique}

22 L'hypothèse souvent avancée est que les airs portent la marque de la prosodie de la langue parce qu'ils sont d'abord créés à partir de mots. Ce sont les mots et, par conséquent, le discours qui donnent le rythme à la musique. Il est possible d'étayer cette proposition de deux manières différentes.

La première est de relier la musique directement au discours. Ceci est particulièrement vrai en Afrique où le lien entre langue et musique est naturel. Au Ghana par exemple, les rythmes ewe $^{3}$ commencent avec des gestes, marqués par le rythme d'un discours libre et se terminent par des gestes stylisés caractérisés par le rythme d'un discours stylisé (Agawu 1995 : 29-30). Klaus Wachsmann (1971: 187) affirme ainsi: «there is hardly any music in Africa that is in some way rooted in speech $»^{4}$. Chernoff $(1979: 75)$ souligne que la musique africaine est dérivée de la langue. Le blues dont certains traits sont hérités de l'Afrique s'inscrit dans ce rapport étroit: on se souvient que des similitudes existent avec la musique africaine. Ce qui a changé, c'est la langue.

Patel (2007 : 165) propose une autre hypothèse, celle de la voie directe (the direct route hypothesis) basée sur l'idée que les schémas prosodiques de la langue maternelle sont acquis dès la petite enfance. En effet, il semble établi que les jeunes enfants sont très sensibles aux schémas prosodiques de leur langue (voir également Nazzi et al. 1998, Ramus 2002). Ils enregistrent de manière statistique, probablement inconsciente, les schémas prosodiques de la langue. C'est ce qu'on appelle « l'apprentissage statistique » («statistical learning ", Patel et al. $2006: 3043$, Patel $2007: 224-225$ ). Ce type d'acquisition des schémas intonatifs ne se limite pas à la seule tendre enfance. On peut supposer que les adultes sont eux aussi sensibles à la musique de la langue et en acquièrent les intonations et le rythme. Ainsi, les esclaves noirs, dont la langue natale n'était certainement pas l'anglais, ont pu s'appuyer sur les schémas rythmiques de la nouvelle langue qu'ils entendaient tous les jours, même sans comprendre. Ils se fiaient à l'image sonore de la langue, au signifiant, pour ensuite inférer les sens des mots et des énoncés. Springer (1999: 54) note ainsi que le « répertoire de morceaux héroïques (selon lui le plus fourni) a été fortement marqué par les ballades du folklore blanc américain, lui- 
même influencé par le fond considérable des ballades anglaises ». Certaines de ces ballades, interprétées par des chanteurs noirs sont d'ailleurs devenues des blues.

Il semble donc plausible que les airs de blues et surtout leur rythme reflètent la prosodie de la langue anglaise telle qu'elle se manifestait à «l'époque dite 'préblues'" (Springer 1999: 54). A cette époque, les esclaves noirs, qui parlaient divers dialectes africains, avaient tendance à calquer le rythme et la syntaxe de l'anglais, leur nouvelle langue, sur leur langue maternelle. «Ce qu'on appelle aujourd'hui l'accent du sud ou le 'parler noir' (jive talk) n'était autrefois que l'accent d'un étranger essayant de parler une langue qu'il ne connaissait pas ». (Jones 1968: 45). Il n'est pas inutile non plus d'insister sur le caractère oral du blues, parfaitement apte à rendre la musique de l'anglais qui en est le véhicule.

Il peut cependant y avoir un conflit entre les exigences musicales et les règles phonologiques de la langue, et lorsque cela se produit ce sont généralement les premières qui l'emportent. C'est pourquoi l'hypothèse de la voie directe qui pose que la prosodie du discours influence nécessairement la structure musicale ne peut être appliquée de manière déterministe (Patel 2007 : 165). Cela veut simplement dire que les chanteurs de blues peuvent ou ont pu être influencés par les schémas rythmiques de l'anglais qu'ils ont engrammés et qu'ils réactivent mentalement. Ces schémas trouvent un reflet dans les textes des chansons, souvent formés de mots usuels, de tous les jours, mono ou dissyllabiques pour la plupart, et énoncés en termes parfois ambigus.

\subsection{L'énoncé dans le blues}

Nous avons vu que le texte dans la tradition africaine était aussi important, sinon plus, que la musique. C'est lui qui, en quelque sorte, dicte le rythme et l'intonation. Les qualités tonales et le timbre de voix d'un chanteur africain ou noir américain en font ressortir les accents et les nuances. Les cris et les hollers, par exemple, n'étaient en définitive que des paroles très rythmées (Jones 1968 : 55).

Dans le blues, comme dans le discours africain, ce n'est pas toujours la régularité qui prime. Le chanteur préfère exprimer sa pensée de manière indirecte, par circonlocution. La simplicité de l'énoncé dénote en effet un manque d'imagination, voire d'intelligence. La même tendance se reflète dans la musique : les notes ne sont pas attaquées franchement, les durées et les accents sont imprécis (cf. Jones 1968 : 59), il est fréquent que le musicien sorte de la tonalité pour faire des digressions; les notes comme les phonèmes - sont tendues, c'est-à-dire qu'elles se caractérisent par une déformation plus grande que leur valeur initiale. On retrouve les longues phrases roulantes et languissantes héritées des field hollers et qui résonnent toujours comme des sanglots, des cris de colère ou de découragement dans le discours. Par exemple, le fameux bluesman Chester Arthur Burnett, alias Howlin' Wolf (1910-1976), définissait le blues dans sa voix forte et grave en ces termes:

(4) I wanna tell you what the blues is. If you ain't got no money, you got the blues. If you ain't got no money you can't buy you a house with, you still got the blues. If you ain't got no money and you can't buy you no food, you got the blues. When you ain't got no money, you got the blues, 'cause you thinkin' evil, you thinkin' 'bout the blues. When you're down, you got the blues. Any time you thinkin' evil, you thinkin' 'bout the blues. (prononcé lors d'un concert en 1966) ${ }^{5}$ 
Cemmentaire ressemble fort à une revendication et pourrait très bien, par sa structure et le rythme de ses phrases, faire l'objet d'un air de blues. Tous les ingrédients y sont : la répétition (reprise de la même phrase if you got no money, you got the blues), la syncope (effacement de l'auxiliaire, ex. you got the blues, you thinkin' evil, des syllabes inaccentués, ex. 'bout, certaines consonnes finales, ex. thinkin') le rythme (l'alternance des syllabes accentuées et des syllabes inaccentuées, ex. you 'got the 'blues), les stéréotypes (l'argent qui permet tout et en particulier de vivre, de se loger et de manger: buy you a house, buy you food, les idées noires: you thinkin' evil). Ce sont les préoccupations de tous les jours qui motivent les thèmes abordés.

\subsection{Les thèmes dans le blues}

Le blues est au départ une ballade, une ballade noire élaborée, on l'a vu, sur le modèle des ballades populaires anglaises et un mode de communication (cf. Herzhaft 2008 : 25). Ce sont des chansons qui racontent les événements de la vie quotidienne. Les journées commencent comme à l'ordinaire : I woke up this morning, feel 'round for my shoes, I woke up this morning I believe I'll dust my broom (En me réveillant ce matin je cherchais mes chaussures, en me réveillant ce matin je croyais que j'allais dépoussiérer mon balai); de nombreux blues s'ouvrent sur ce type de phrase. Ensuite, les choses se compliquent un peu avec les problèmes de la vie amoureuse et conjugale : Before you accuse me, take a look at yourself (Avant de m'accuser, regarde-toi bien), l'abandon : One summer day she went away, she gone and left me (Un jour d'été, elle est partie et m'a laissé), la rupture : I'll pack up my trunk, and make my get away (Je vais faire ma valise et partir) et l'alcool, très présent : You're a whiskey-headed woman, you stay drunk all the time (Tu es une femme qui ne pense qu'au whiskey, tu es ivre tout le temps). Le thème du train que les Noirs empruntaient pour se déplacer apparaît souvent: Gonna leave on the Sunshine Special, gonna leave on the Santa Fe (Je vais partir avec le Sunshine Special, je vais partir avec le Santa Fe). Pour finir, on oublie ses ennuis, on se sent libéré : I can't worry, 'cause I'm sitting on the top of the world (Je ne m'en fais pas, parce que je suis aux anges).

Sorte de ménestrels de la population noire, les chanteurs de blues relataient souvent les événements locaux comme la crue du Mississippi (Rising High Water Blues) ou la réparation des digues rompues (Broken Levee Blues). On parle des villes alentour, Cairo (Géorgie), Helena (Alabama), Memphis (Tennessee), la Nouvelle Orléans (Louisiane), Saint-Louis (Missouri), et des lieux plus lointains qui représentent l'émancipation et la prospérité, le voyage comme Chicago ou la Californie. Mais le blues a aussi une dimension poétique, ce qui signifie que la langue vise à suggérer, à créer des images, des sens cachés, signe d'une liberté d'expression réprimée.

\subsection{Le second degré}

Quand on lit ou qu'on écoute un texte de blues, la méfiance est de mise. L'énoncé n'est pas univoque, le message étant bien souvent codé. Le biais des allusions sexuelles, les grivoiseries, les expressions argotiques, les sous-entendus, les mots à double sens sont légion, d'où les multiples interprétations possibles (y compris les contresens) d'un énoncé. Ceci est à rapprocher du caractère indirect de l'énoncé africain, le chanteur de blues jouant avec les mots, le rythme et la musique de la langue. Dans l'Amérique puritaine de l'époque, il aurait été malvenu de chanter des chansons grivoises, ou de 
parler de sexe. Les chanteurs utilisaient donc souvent la technique du double sens pour faire passer ce type de message. Ainsi, l'expression I got my mojo working peut se lire de deux façons différentes. Au premier degré, l'énoncé signifie : j'ai mon mojo qui marche, le mojo étant une sorte de petit sac, une amulette aux vertus magiques utilisée en vaudou, et par conséquent lié aux superstitions des esclaves noirs. Prise au second degré, l'expression renvoie à la puissance sexuelle. Un autre exemple de double sens à connotation sexuelle se cache derrière l'énoncé I believe I'll dust my broom. Au premier degré, on peut naïvement lire : « je crois que je vais dépoussiérer mon balai », mais avec un peu d'imagination on entrevoit sans grande difficulté l'allusion sexuelle, et le symbole phallique du balai. Ce type d'allusion se glisse jusque dans l'expression rock and roll qui fait évidemment référence à être bercé et par extension à une danse, mais dont le sens second renvoie à l'acte sexuel (cf. Rock me baby, rock me all night long ... Roll me baby, like you roll a wagon wheel, B.B. King).

Le symbolisme n'est pas seulement sexuel bien sûr. Dans la chanson Sweet Home Chicago, le chanteur (Robert Johnson) évoque son rêve d'aller vers la Californie (back to the land of California to my sweet home Chicago). A première vue, il y a une contradiction, une erreur géographique, mais ici il ne s'agit pas de la Californie en tant qu'état, mais de ce qu'elle représente métaphoriquement, le pays de la richesse et de la liberté, c'est-à-dire la ville de Chicago pour un chanteur de blues pauvre et misérable du Mississippi.

Enfin, au-delà des doubles sens et des contraires, il y a la religion, plus précisément la place réservée à Dieu et au diable. On se souvient que le blues et les negro spirituals sont deux aspects d'une même tradition, l'un est profane, l'autre sacré. Si les negro spirituals parlent de Dieu, le blues ne l'évoque guère. Souvent qualifié de diabolique, sûrement à cause des débordements vocaux (cris, hurlements de désespoir, plaintes, etc.) qui le caractérisent, de ses racines africaines, des thèmes qu'il développe et surtout des allusions grivoises déguisées, mais aussi parfois clairement et grossièrement exprimées (notamment dans le fameux Shave 'em Dry chanté par Lucille Bogan qui décrit crûment les choses du sexe), le blues est ce qu'on pourrait appeler la contrepartie profane du negro spiritual. On a pourtant l'impression que le diable et le blues font bon ménage. Ils représentent tous les deux ce qui ne va pas, ce qui est mal (cf. Howlin' Wolf : When you thinkin' evil, you thinkin' 'bout the blues, " quand tu penses au mal, tu penses au blues »). Symboliquement, le diable renvoie au méchant, à l'obscurité et à la nuit (et en cela, il s'oppose à Dieu, centre de lumière [Dianteill 2004 : 428]), aux problèmes, aux choses négatives, aux forces qui troublent et affaiblissent la conscience (Chevalier et Gheerbrant 1969 : 352), comme la femme, par exemple (Devil Got my Woman, Skip James, She Belongs to the Devil, Washboard Sam). Dans certains airs, le chanteur semble avoir passé un pacte avec le diable: Me and the devil were walkin' side by side (Robert Johnson), Early this morning, when you knocked upon my door/And I say hello Satan, I believe it's time to go (Gil Scott Heron). Parfois, c'est le diable lui-même qui est déprimé et triste (Devil's got the blues, Lonnie Johnson), quand les blue devils deviennent le devil's blues (le blues du diable). La boucle est ainsi bouclée.

Le blues est pour ainsi dire une réaction cathartique, en ce sens qu'en même temps qu'il permet de soulager les peines et les blessures de la vie, il représente une libération à la fois individuelle et collective d'émotions socialement inacceptables. Il s'agit de dire tout haut, par des formulations certes déviantes par rapport à la langue courante (ce qui contribue, on l'a vu, à classer les locuteurs dans une catégorie sociale inférieure), d'extérioriser ce qu'on a longtemps refoulé, de dénoncer des conditions d'existence 
déplorables et parfois inhumaines, de montrer à la société américaine sa face cachée, honteuse, sombre et cruelle, pour ne pas dire infernale. Ce contexte met en évidence le rôle social de la langue, cette dernière étant un moyen de communication entre les individus fondé sur le même système conceptuel. Ceci suppose la création d'un certain nombre de préceptes auxquels les utilisateurs de la langue doivent se conformer. Ces règles définissent une pratique normative des rapports sociaux d'ordre hiérarchique. Dans ce sens, l'acceptabilité d'un énoncé se rapporte directement à un modèle de référence unique (en l'occurrence celui des maîtres) qui mène tout naturellement à l'adoption et à la reconnaissance de la langue dominante.

Cependant, les jugements que l'on porte sur la langue sont en grande partie liés au rapport qui existe entre le social et l'utilisation de la langue et ne concerne pas toujours le message.

\section{Le rôle social du blues}

Outre le rôle culturel, informationnel et poétique du blues, il est clair que ce genre musical a aussi une fonction, ou plutôt des fonctions, sociales celles-là (Springer 1999), qui ont permis au groupe d'exister et de grandir malgré les difficultés, les contraintes, les humiliations et les interdits de toutes sortes, que ce soit dans le travail, au niveau religieux ou dans l'expression corporelle.

\subsection{Le travail}

Les esclaves noirs importés d'Afrique étaient considérés comme de véritables bêtes de somme. Pour survivre, ils devaient être capables et performants et servir le maître du mieux possible. Les chants en appels et répons qui accompagnaient et rythmaient le travail des champs et plus tard le travail des chaingangs ${ }^{6}$, venaient tout droit des traditions d'Afrique de l'ouest (Herzhaft 2008 : 15). Ce faisant, ces chants traditionnels venus d'Afrique ont subi une évolution, un transfert linguistique et rythmique (Jones 1968 : 45). Peut-être ici dirons-nous que le chant africain a influencé l'anglais dans sa syntaxe et dans son rythme.

Chanter pendant le travail remplissait deux fonctions essentielles. La première est de rythmer l'effort de travail pour encourager le travailleur à avancer dans sa tâche, à l'aider à supporter sa condition. Un esclave noir qui travaille mal risque d'être sanctionné sévèrement ou vendu. De plus, cela donnait aux maitres une impression de joie de vivre et de bonheur (Herzhaft 2008: 14). La seconde fonction est la prise de conscience du groupe: chanter ensemble avec un soliste et un chœur qui répond facilite la cohésion, et la musique en est un des ciments (Patel $2007: 370$ ). Rappelons qu'il s'agit d'une langue nouvelle, donc d'une musique nouvelle, qu'il faut apprendre coûte que coûte. En outre, le fait de faire de la musique en groupe, non seulement améliore l'humeur, mais aussi resserre les liens entre les membres du groupe. La musique, comme la langue, est un facteur de cohésion sociale.

D'autre part, les mères qui travaillaient aux champs pouvaient ainsi garder un lien rassurant avec leurs bébés sans pour autant les toucher (Balter 2004), le chant constituant ce moyen de contact (Patel 2007 : 370, Balter 2004, Falk 2004). Les enfants pouvaient ainsi être sensibilisés à la musique de la langue, à sa mélodie et à son rythme, comme nous le montrerons plus loin. Les mères possédaient toutefois d'autres moyens 
pour réconforter leurs jeunes enfants, le chant n'étant qu'un moyen parmi d'autres. En revanche, celui-ci était bel et bien l'instrument privilégié de l'expression sacrée.

\subsection{La fonction religieuse}

41 Selon Chevalier et Gheerbrant, "Le chant est le symbole de la parole qui relie la puissance créatrice à sa création en tant que celle-ci reconnait sa dépendance de créature et l'exprime dans la joie, l'adoration ou l'imploration. C'est le souffle de la créature répondant au souffle créateur » $(1969$ : 206). Pour parler à Dieu, l'homme doit chanter, et ce faisant il se met sous sa domination, sa protection, il accepte sa supériorité. Une autre forme de rapport hiérarchique s'instaure ainsi pour l'homme noir : l'esclave est soumis d'une part au maître qui l'opprime, et d'autre part à Dieu qui lui donne la joie.

42 La société des planteurs esclavagistes était résolument chrétienne et pratiquante. Après avoir considéré les esclaves comme des bêtes, on se mit à les évangéliser en masse (Herzhaft 2008: 15) : d'une part, pour tenter d'effacer complètement la religion de l'homme africain; d'autre part, afin de leur consentir un minimum d'instruction religieuse leur permettant de passer du statut de païens ou de sauvages au statut d'être humain civilisé à part entière. Ce processus constituait une manière d'intégration: même s'ils restaient des esclaves, ils devenaient américains (cf. Jones 1968 : 66-67). Qui plus est, on leur apportait une compensation et le bonheur de la foi en un Christ blanc tout puissant dont l'influence était supérieure aux dieux protecteurs des religions d'origine africaine.

43 En Afrique, la pratique religieuse ne se faisait pas sans chant (Jones $1968: 73$ ) et cette tradition s'est étendue à la culture noire américaine. Par conséquent, le chant religieux chrétien est devenu un autre moyen d'expression privilégié. On se souvient que les esclaves noirs adaptaient et modelaient les hymnes et les cantiques baptistes et méthodistes en chants rythmés, mêlant les origines africaines et européennes que l'on connaît sous le nom de negro spirituals ou gospel. On notera au passage le parallèle établi entre les souffrances du peuple juif opprimé de l'Ancien Testament et celles des esclaves noirs qui vont pour ainsi dire s'identifier au peuple de Moïse fuyant l'Égypte vers la liberté, et pour les Noirs la Terre Promise de l'émancipation (Jones 1968: 82, Herzhaft 2008 : 16). "Traverser le Jourdain ", par exemple, signifiait à la fois aller au paradis et rejoindre le Christ, et d'une certaine façon se libérer de ses souffrances terrestres et devenir des citoyens libres et égaux dans la société américaine.

Contrairement aux work songs, les chants religieux n'étaient pas des cris ou des plaintes. La musique était plus douce, plus mélodique, même si le cri apparaît encore de temps en temps comme une survivance africaine. Mais elle prend aussi la forme d'une expression corporelle quand l'émotion devient trop forte et conduit quelquefois à des transes extatiques, au sens de danse frénétique qui se termine souvent par une perte de connaissance (Dianteill 2004: 437). On commence par frapper ses mains l'une contre l'autre pour marquer la cadence, puis on se balance à mesure que l'intensité monte, enfin on se laisse gagner par le mouvement de la musique. Dans ce cas, c'est le rythme qui prend le dessus, le rythme des chants, le rythme des paroles, le rythme de la langue. 


\subsection{La danse}

la vie de tous les jours. Dans les familles qui vivaient sur les plantations, le samedi soir était souvent consacré aux chants et aux danses. Les danses étaient un mélange de figures diverses d'origine africaine et européenne qui allait donner la "plantation dance », la danse des plantations (Herzhaft 2008 : 18). Par cette danse, les esclaves exprimaient leurs émotions, leurs ressentis. Ils tapaient du pied, tournaient à contresens des aiguilles d'une montre, se frappaient les bras, les jambes, la poitrine et les joues. La danse des plantations, aussi appelée juba dance, était pratiquée sans instrument rythmique : les maîtres craignaient en effet que les tambours pussent être utilisés pour transmettre des codes et des messages secrets et les interdisaient, et ce n'est qu'au milieu du XIXe siècle que de la musique et des paroles furent ajoutées. Parmi les danses issues de la plantation dance, on retiendra le jump Jim Crow, danse préférée des fameux minstrel shows ${ }^{7}$, qui fait allusion au système " Jim Crow » dans les états du sud. Jim $\mathrm{Crow}^{8}$ était synonyme de racisme et de ségrégation ${ }^{9}$. Ce type de danse a sans doute aussi influencé la danse à claquettes (tap dance), d'origine africaine et européenne (irlandaise), dont le rythme syncopé fait alterner des temps forts et des temps moins marqués.

\section{Conclusion}

Il est important de retracer l'histoire du blues, ses origines à la fois africaines et européennes (américaines), sa langue qui a évolué au cours des derniers siècles et qui s'est imposée comme une variété de l'anglais américain. Certains Blancs du sud des Etats-Unis ont d'ailleurs adopté, du moins dans son accent et parfois dans son accentuation, cette langue qui a été un facteur important de cohésion et d'intégration du peuple noir.

Comme on l'a vu, le blues possède deux facettes, l'une sacrée, l'autre profane, l'une est celle du bon Dieu avec ses prières et ses espérances, l'autre celle du diable avec ses cris, ses plaintes et ses références au sexe. C'est dans la langue que se manifestent ces deux aspects. Rappelons que la langue est constituée par les mots et le discours mais aussi par l'intonation et le rythme, et ce poids linguistique a certainement marqué la musique des Afro-Américains.

La musique africaine est directement dérivée de la langue et l'on peut légitimement dire que certains traits musicaux du blues présentent de nombreux points communs 
avec la langue anglaise. Mais il y a aussi l'hypothèse de l'apprentissage statistique, à savoir la recherche de schémas prosodiques dans l'environnement et l'acquisition des connaissances implicites sans rétroaction directe. C'est d'abord l'image sonore de la langue qui guide les locuteurs potentiels dans leur appropriation de la langue (qu'ils soient enfants ou adultes), image qui se retrouve dans les chants repris en chœur dans les work songs, field hollers et negro spirituals.

Ainsi, l'énoncé dans le blues, comme dans la tradition africaine, conditionne en quelque sorte le rythme et l'intonation. En principe, le chanteur de blues primitif ou rural n'a pas étudié la musique, c'est un musicien autodidacte et pour ainsi dire routinier. C'est un chemineau démuni et analphabète, parfois aveugle - on songe ici à Blind Lemmon Jefferson, Blind Blake, Blind Boy Fuller, Blind John Davis - qui ressemble au personnage évoqué dans le roman Sartoris de William Faulkner :

Against the wall, squatting, a blind Negro beggar, with a guitar and a wire frame holding a mouth-organ to his slips, patterned the background of smells and sounds with a plaintive reiteration of rich, monotonous chords, rhythmic as a mathematical formula, but without music. He was a man of at least forty and his was that patient resignation of many sightless years... (108-109) ${ }^{10}$

On retrouve dans cet extrait l'idée de régularité et de conformité à un modèle, un usage, une tradition. La monotonie de la musique traduit la résignation du personnage à sa condition.

Sur les plantations, la musique et les chants sont fréquemment accompagnés de danse. La danse est largement utilisée, bien sûr, comme mode de divertissement, mais aussi comme vecteur d'information et de perpétuation des cultures et des traditions. Nous venons de voir que certaines danses, notamment le juba dance, comportaient des gestes signifiants aptes à traduire la joie, la peine, la colère, la peur, la surprise, la révolte, bref à véhiculer un message. Selon Dianteill (2004: 437), « en observant la façon de danser d'une personne, on pouvait déceler quel était l'esprit qui l'inspirait». On songe évidemment au diable ou au bon Dieu, ou encore à des esprits païens qu'on invoque par superstition.

Le blues propose un message volontairement et fréquemment ambigu (doubles sens, allusions grivoises de toutes sortes, métaphores, sous-entendus, etc., souvent associés au diable). En principe, ce n'est pas véritablement une musique de danse. Le rythme des blues primitifs est trop lent et les instruments qui l'accompagnent sont en nombre restreint. C'est la voix du chanteur et son timbre qui sont mis en évidence. Il s'agit, en effet, de faire passer un message, de faire comprendre ce que l'on chante. En réalité, « le blues est un discours autant qu'une musique » (Dianteill 2004 : 423), un discours qui n'oublie jamais de rappeler l'enfer vécu par la population afro-américaine.

Agawu, K. (1995) African Rhythm: A Northern Ewe Perspective. Cambridge: Cambridge University Press.

Attridge, D. (1982) The Rhythms of English Poetry. London \& New York: Longman.

Balter, M. (2004) « Seeking the key to music. » In Science, 306: 1120-1122.

Bolinger, D. (1985) Intonation and its parts: Melody in Spoken English. London: Edward Arnold.

Carr, P. (1999) English Phonetics and Phonology. An Introduction. Oxford: Blackwell. Chernoff, J. (1979) African Rhythm and African Sensibility. Chicago: Chicago University Press.

Chevalier, J. et A. Gheerbrant (1969) Dictionnaire des symboles. Paris : Robert Laffont. 
Dianteill, E. (2004) « La danse du diable et du bon dieu. Le blues, le Gospel et les églises spirituelles.) Editions du EHESS. L'Homme 2004/3-4, n 171-172, p. 424-441.

Falk, D. (2004) « The 'putting the baby down' hypothesis: Bipedalism, babbling, and baby slings. » In Behavioral and Brain Sciences, 27: 526-534.

Faulkner, W. (1925) Soldier's Pay. London: Picador.

Faulkner, W. (1953) Sartoris. New York: New American Library.

Forten, C. (1961) A Free Negro in the Slave Era. New York: Macmillan.

Herzhaft, G. (2008) Le Blues, $5^{\mathrm{e}}$ édition. Que sais-je ? Paris : P.U.F.

Jones, L. (1968) Le Peuple du blues. Titre original: Blues People (1963). Paris: Gallimard. Ker, W.P. (1928) Form and Style in Poetry: Lectures and Notes. London: Chambers. Levet, J.P. (1992) Talkin' that talk. Le langage du blues et du jazz. Paris: Nathan.

Nazzi, T., J. Bertoncini \& J. Mehler (1998) « Language discrimination by newborns.

Toward an understanding of the role of rhythm. » In Journal of Experimental Psychology: Human Perception and Performance, 24: 756-777.

Patel, A.D. (2007) Music, Language, and the Brain. Oxford: Oxford University Press.

Patel, A.D., J.R. Iversen, et J.C. Rosenberg (2006) « Comparing the rhythm and melody of speech and music. The case of British and French. » In Journal of the Acoustical Society of America, 119: 3034-3047.

Ramus, F. (2002) « Language discrimination in newborns: Teasing apart phonotactic, rhythmic, and intonational cues. " In Annual Review of Language Acquisition, 2 : 85-115. Springer, R. (1999) Les fonctions sociales du blues. Marseille : Parenthèses.

Wachsman, K. (1979) Essays on Music and History in Africa. R.P. Wachsman (ed.). Evanston: Northwestern University Press.

Annexe

Traduction du passage tiré de Soldier's Pay de W. Faulkner : ${ }^{11}$

... Le chemin replongeait entre les balafres rougeâtres de la terre et, d'un espace éclairé de lune, interrompu par un bouquet d'arbustes, leur parvenait une musique, pure, vibrante, sans paroles et lointaine.

«Ils célèbrent leur messe. Les Noirs », a expliqué le recteur. Ils poursuivirent leur route dans la poussière, dépassant de petites maisons nettes, bien rangées, assombries par le sommeil. Un groupe de Noirs les dépassa, ils portaient des lanternes allumées qui projetaient de vaines petites flammes, futilement, dans le clair de lune. «Personne ne sait pourquoi ils font ça, répondit le recteur à la question de Gillian. Peut-être pour éclairer leur église. »

Le chant se rapprochait toujours plus. Finalement, en s'accroupissant au milieu d'un bouquet d'arbres sur le bord de la route, ils aperçurent l'église miteuse. A l'intérieur, il y avait une douce lueur de pétrole qui ne faisait que rendre l'obscurité et la chaleur plus épaisses. Plus épaisse aussi était l'imminence du sexe après une rude journée de labeur sur la terre baignée de lune, de laquelle le chant immergé de passion de la race noire montait. Ce n'était rien et c'était tout. Puis il a enflé jusqu'à l'extase, empruntant les paroles de l'homme blanc aussi allègrement qu'il s'était approprié son Dieu lointain. 
Nourris ce troupeau, ô Jésus. Tout ce désir qu'a l'humanité de s'unir à quelque chose, quelque part. Nourris ce troupeau, Ô Jésus...

Le recteur et Gillian se tenaient côte à côte sur le chemin de terre. Celui-ci s'éloignait sous la lune. Se dissolvait vaguement à perte de vue. Les champs usés et creusés de sillons rouges n'étaient désormais que des taches alternées de noir léger et d'argent ; les arbres avaient chacun un halo argenté, sauf ceux qui, protégés de la lune, semblaient coulés dans le bronze.

Nourris ce troupeau, ô Jésus. Les voix s'élevaient pleines et douces. Il n'y avait pas d'orgue ; aucun orgue n'était nécessaire. Au-dessus des harmonies passionnées des basses et des barytons s'élevait le soprano clair des voix de femmes comme une envolée d'oiseaux célestes et dorés. Ils restèrent plantés là, dans la poussière, le recteur dans sa tenue noire informe, et Gillian dans la sienne en serge toute neuve et amidonnée, écoutant et voyant l'église minable transfigurée par son aspiration tranquille, triste et enflammée. Puis les chants cessèrent, s'estompant sur la terre baignée de lune, avant que ne viennent le lendemain, la sueur, le sexe, la mort et la damnation inévitables. Puis ils prirent la direction de la ville sous la lune en sentant la terre dans leurs souliers.

\section{NOTES}

1. Voir traduction en annexe.

2. A Free Negro in the Slave Era (1961).

3. Peuple du Togo et du Ghana en Afrique occidentale.

4. «Il n'y a pratiquement aucune musique en Afrique qui d'une manière ou d'une autre ne vienne du discours.»

5. «Je vais te dire ce qu'est le blues. Si t'as pas d'argent, t'as le blues. Si t'as pas d'argent pour te payer une maison, $t^{\prime}$ as encore le blues. $S i t^{\prime}$ as pas d'argent et que tu peux pas t'payer de quoi manger, $t^{\prime}$ as le blues. Quand $t^{\prime}$ as pas d'argent, $t^{\prime}$ as le blues, pa'ce que tu penses au mal, tu penses au blues. Quand t'as l'cafard, t'as le blues. Chaque fois que tu penses au mal, tu penses au blues ». Notre traduction.

6. Groupes de forçats enchaînés ensemble, en particulier pendant le travail en extérieur.

7. Créés au début et au milieu du XIXe siècle aux USA, les minstrel shows étaient des spectacles populaires composés de dialogues comiques, de chansons et de danses, interprétés d'abord par des troupes d'acteurs blancs qui se noircissaient le visage, puis, surtout après la guerre de sécession, par des comédiens noirs. Ces spectacles visaient à caricaturer les noirs, à imiter leur apparence et leur façon de chanter.

8. Du nom d'une chanson interprétée par Thomas D. Rice (1808-1860), premier comédien blanc à se noircir le visage, dans un minstrel show.

9. On pense aux lois Jim Crow qui institutionnalisèrent la ségrégation raciale dans les états du sud des Etats-Unis.

10. «Contre le mur, accroupi, un mendiant noir aveugle, avec une guitare et une monture en fil de fer qui lui maintenait aux lèvres un harmonica, plaquait sur cet arrière-plan d'odeurs et de 
bruits la plainte réitérée d'accords chauds et monotones, aussi rythmés qu'une formule mathématique, mais sans musique. C'était un homme d'au moins quarante ans et il avait cette patience résignée que l'on a après de nombreuses années de cécité. » Notre traduction.

11. Notre traduction.

\section{RÉSUMÉS}

Le blues est une forme musicale traditionnelle dérivée des chants de travail des anciens esclaves. Il semble que le terme «blues " provienne de l'expression anglaise « the blue devils» qui signifie idées noires, dépression et tristesse. On en retrouve une référence dans une pièce de théâtre en un acte de Georges Coleman le Jeune, Blue Devils, a Farce in One Act (1736).

Le blues est, au début, une ballade narrative inspirée de la musique folklorique venue d'Europe et un mode de communication. Ce sont des airs qui racontent les malheurs personnels vécus par les Afro-Américains. Ces chants ont une dimension poétique, l'énoncé n'est pas univoque : bien souvent, le message est codé et abonde en mots à double sens, allusions sexuelles, grivoiseries, expressions argotiques, sous-entendus, métaphores, etc. A l'origine du blues, on trouve aussi les negro spirituals qui laissent une large place à Dieu (la lumière céleste) et au diable (le mal et les ténèbres) qui semblent faire bon ménage. Dans certains airs, le chanteur a conclu un pacte avec le diable; c'est parfois le diable lui-même qui est déprimé et triste. Quand les blue devils deviennent le devil's blues (le blues du diable), la boucle est bouclée.

Outre le rôle culturel et poétique du blues, ce genre musical a des fonctions sociales qui ont permis à la communauté de survivre et de grandir malgré les difficultés et les contraintes.

Blues is a traditional musical form originated by former slaves, and which developed from work songs and field hollers. The term "blues" may have come from the blue devils, describing a depressed mood; an early use of the phrase in this sense occurs in George Colman the Younger's one-act play Blue Devils, a Farce in One Act (1736).

At the beginning, blues is simple narrative ballads inspired by folk music from Europe, and a mode of communication. The songs usually relate troubles and personal woes experienced by African American people. There is a poetic dimension that is present in these songs, the verse is not univocal and the message is often expressed in an indirect way, rife with wordplay and double meanings: for example, sexual allusions, saucy expressions, slang, innuendoes, metaphors, etc.

Blues also includes spirituals, saving an important place for God (the holy light) and the devil (evil and darkness), who seem to get along quite well. In some songs, the singer has made a pact with the devil; sometimes, the devil himself has got the blues. When the blue devils become the devil's blues, things come full circle.

Besides the cultural and poetic role of blues, this musical genre has social functions which have enabled the community to survive and grow up despite difficulties and constraints, at work, in their faith, and in their self-expression. 
INDEX

Thèmes : Music

Keywords : blues, song, danse, negro spiritual, prosody, rhythm, work songs

Mots-clés : blues, chant, danse, negro spiritual, prosodie, rythme, work songs

\section{AUTEURS}

\section{PATRICE LARROQUE}

Professeur à l'Université Toulouse-Jean Jaurès

patrice.larroque@univ-tlse2.fr 